\title{
Incidencia de la agrupación de los jugadores en el diseño de las tareas de entrenamiento en balonmano Impact of players' grouping on the design of handball training tasks
}

David Mancha-Triguero, Belén Baquero, Sergio J. Ibáñez, Antonio Antúnez

Universidad de Extremadura (España)

\begin{abstract}
Resumen. El ciclo de calidad de la intervención del entrenador implica conocer como es el proceso de planificación y su aplicación práctica, para posteriormente proceder a su análisis y evaluación. El principal objetivo de esta investigación fue caracterizar el proceso de entrenamiento que realiza un equipo amateur masculino de balonmano y la relación que existe entre la variable Situación de Juego con en el resto de las variables que conforman el entrenamiento. Para ello, las situaciones de juego se agruparon en situaciones sin oposición, situaciones individuales, situaciones reducidas de igualdad numérica, situaciones reducidas de superioridad numérica y juego completo. Se analizaron un total de 141 tareas de entrenamientos y se codificaron a través del instrumento Sistema Integral para el Análisis de las Tareas de Entrenamiento. Se realizó un análisis descriptivo de las Variables Pedagógicas y de Carga Externa para posteriormente estudiar la relación entre estas variables con la variable independiente Situación de Juego. Para ello, se utilizaron las pruebas estadísticas $\mathrm{Chi}^{2}$ para la relación de dependencia y $V$ de Cramer para calcular el nivel de asociación a través de los Residuos Tipificados Corregidos. El análisis confirma que el entrenador emplea situaciones reducidas y se muestran diferencias en todas las variables analizadas en función delTipo de Situación de Juego. Una mayor variedad en el empleo de las diferentes variables que definen una tarea facilita y fomenta el desarrollo pedagógico y físico del jugador del balonmano que repercute en el resultado final en competición.
\end{abstract}

Palabras Claves: Deporte de Equipo, Análisis de Tareas, Sistema Integral para el Análisis de las Tareas de Entrenamiento, Situación de Juego, Entrenador.

Abstract. The quality cycle of the coach's intervention implies knowing what the planning process and its implementation are like, in order to subsequently proceed to its analysis and evaluation. The purpose of this research was to describe the training process carried out by a male amateur handball team and how the Game Situation variable influences the rest of the variables that make up the training. To do this, the game situations were grouped into Unopposed Situations, Individual Situations, Small Sided Games of Numerical Equality, Small Sided Games of Numerical Superiority and Full Games. A total of 141 training tasks were analyzed and coded using the Integral Analysis System of Training Tasks. A descriptive analysis of the Pedagogical and External Load Variables was carried out to later study the relationship between these variables with the independent variable Game Situation. For this, the $C h i^{2}$ statistical tests for the dependency relationship and $C r a m e r$ 's $V$ were used to calculate the level of association through the Corrected Typified Residuals. The analysis confirms that the coach uses reduced situations and differences are shown in all the variables analyzed depending on the Type of Game Situation. A greater variety in the use of the different variables that define a task facilitates and encourages the pedagogical and physical development of the handball player, which affects the final result in competition.

Keywords: Team Sport, Task Analysis, Integral System for Analysis of Training Tasks, Game Situation, Coach.

\section{Introducción}

El balonmano es uno de los deportes de invasión con mayor repercusión a nivel nacional y mundial (Consejo Superior de Deportes, 2019). Está integrado dentro de los deportes de cooperación-oposición con espacio común y participación simultánea sobre el móvil del juego (Hernández-Moreno, 2004). La cooperación consiste en la utilización por parte de los integrantes de un

Fecha recepción: 07-04-21. Fecha de aceptación: 24-06-21

Sergio J. Ibáñez

sibanez@unex.es equipo, de los diferentes medios técnicos y tácticos para la consecución de un objetivo común. Por su parte, la oposición está determinada por la rivalidad del equipo adversario que utilizará sus medios para oponerse a la consecución del objetivo común del contrario (Oliver, 2018).

Las competiciones tanto a nivel nacional como internacional se organizan en función de la edad de los participantes tanto en categoría masculina como femenina. Estas diferencias naturales entre los jugadores repercuten en el diseño y planificación de las sesiones de entrenamiento, puesto que el entrenador debe diseñar el proceso de enseñanza-aprendizaje en función de las 
características de sus jugadores y el momento evolutivo en el que se encuentran (Bompa, \& Buzzichelli, 2018; Cañadas, Gómez, García-Rubio, \& Ibáñez, 2018). Este proceso va a estar influenciado tanto por la metodología del entrenador como por su planificación, que provocará una fusión entre las experiencias previas obtenidas y la formación tanto académica como federativa que tiene (Clemente, Martins, \& Mendes, 2015).

El ciclo de calidad de la intervención del entrenador (Ibáñez, 2009), implica conocer como es el proceso de planificación y su implementación, para posteriormente proceder a su análisis y evaluación. Una de las líneas de investigación académica española más empleadas sobre el entrenador es el de su comportamiento, destacando los tópicos de su comportamiento práctico y su estilo de entrenamiento (Ibáñez, García-Rubio, Antúnez y Feu, 2019). Para la planificación del entrenamiento existen diferentes instrumentos gratuitos o de pago que facilitan la labor del entrenador y su empleo varía en función de los recursos del club o institución en la que se trabaje. Para el control del entrenamiento no existen muchas herramientas que faciliten esta labor. Uno de los instrumentos más empleados en los últimos años es el Sistema Integral para el Análisis de las Tareas de Entrenamiento, SIATE (Ibáñez, Feu, \& Cañadas, 2016), un instrumento que se caracteriza por ser de acceso gratuito y aportar un conocimiento integral sobre el proceso llevado a cabo por el entrenador. Una de las variables que analiza este instrumento es la Situación de Juego (en adelante, SJ). Esta variable se entiende como el número de jugadores que se agrupan para cada tarea (1vs1, 2vs2, etc.). El numero de jugadores durante la tarea puede ser fijo o cambiante. Atendiendo al sistema de clasificación propuesto, la complejidad aumenta directamente proporcional con el incremento de jugadores que se agrupan (Ibáñez et al., 2016).

En Balonmano, al igual que en el resto de los deportes de invasión, existe una corriente sobre el empleo de situaciones reducidas o Small Sided Games (en adelante, SSG). Este tipo de agrupaciones se caracterizan por representar a menor escala las necesidades que el juego completo, Full Games (en adelante, FG) pero con menor número de jugadores, facilitando la asimilación del apartado pedagógico y técnico-táctico (González-Espinosa, Ibáñez, \& Feu, 2017). Además, Hill-Haas, Coutts, Rowsell y Dawson (2009) confirmaron que el empleo SSG favorecen el desarrollo físico del deportista puesto que la participación es mayor y, por tanto, los requerimientos también son más elevados que las situaciones de FG. Esto se explica mediante el análisis de la fre- cuencia cardíaca, identificando que en los jugadores eran más elevadas en los SSG que en los FG (Hill-Haas et al., 2009).

El análisis de los procesos de entrenamiento es una práctica común en deportes como el fútbol (GarcíaCeberino, Gamero, González-Espinosa, Garcia-Rubio, \& Feu, 2018; García-Ceberino, Antúnez, Feu, \& Ibáñez, 2020; Urbano-Arevalo, Mancha-Triguero, GomezCarmona, \& Gamonales, 2020) o el baloncesto (Cañadas, Ibáñez, \& Leite, 2015; Mancha-Triguero, GarcíaCeberino, Antúnez, \& García-Rubio, 2018; GonzálezEspinosa, Mancha-Triguero, García-Santos, Feu, \& Ibáñez, 2019). En balonmano los estudios sobre el control del entrenamiento se han focalizado en el contexto escolar. Gamero, García-Ceberino, González-Espinosa, Reina, \& Antúnez (2017) analizaron las unidades didácticas de los profesores para la enseñanza del balonmano. Por su parte García-Ceberino et al. (2018) evaluaron la carga externa provocada por los programas de enseñanza del balonmano escolar.

El análisis y control de la carga en balonmano no está tan desarrollada en los equipos de formación. Apenas existen documentos públicos en los que se realicen este trabajo de cuantificación de carga de entrenamientos y control de las tareas de entrenamiento en categorías amateur o de formación (Fox, Stanton, Sargent, Wintour, \& Scanlan, 2018). En cuanto a equipos de alto rendimiento o selecciones nacionales, esta práctica es más común, puesto que la mayoría de los instrumentos tienen un alto coste económico (Cummins, Orr, O’Connor, \& West, 2013) y la formación de los entrenadores es mas completa.

Existen pocas evidencias en la literatura que muestren como son los procesos de entrenamiento de equipos de balonmano. Son más aún escasas las referencias que abordan el estudio del entrenamiento en equipos amateur o en periodos de formación. Es necesario incrementar el conocimiento científico de lo que ocurre en estos niveles competitivos para aportar evidencias científicas que ayuden a mejorar los procesos de entrenamiento en balonmano, mejorando la intervención del entrenador y por extensión, la formación de los jugadores. Por todo ello, el objetivo principal de esta investigación fue caracterizar el proceso de entrenamiento que realiza un equipo amateur masculino de balonmano y la relación que existe entre la variable Situación de Juego con en el resto de las variables que conforman el entrenamiento. Se hipotetiza que la SJ influye en las demás variables que definen una tarea de entrenamiento y que el entrenador cuando emplea una determinada agrupa- 
ción el patrón para seleccionar o agrupar el resto de variables es similar.

\section{Método}

\section{Diseño}

El diseño de la investigación se clasificó bajo la descripción de estudio empírico realizado con metodología cuantitativa, descriptiva mediante un código observacional diseñado exprofeso, natural, y retrospectivo (Montero y León, 2007). La obtención de los datos se realizó de manera ecológica y no se interfirió en el diseño de las tareas que planificaba el entrenador.

\section{Participantes}

Se analizó a un entrenador con ocho años de experiencia en deporte base y 12 años de experiencia con equipos senior. Su formación académica era Maestro de Educación Física y es entrenador Nivel II de balonmano. El equipo analizado era un equipo de categoría Senior Masculino ( $n=18$ jugadores) que competía en $2^{\text {a }}$ División Nacional de carácter regional. Los jugadores tenían una edad media de 26 años con una experiencia en el deporte de 11,83 $\pm 1,13$ años, un peso medio de 80 $\pm 10,90 \mathrm{~kg}$, altura media $180 \pm 7,86 \mathrm{~cm}$.

\section{Muestra}

La muestra inicialmente estuvo formada por 141 unidades de análisis estadístico, tareas, diseñadas por el entrenador y realizadas durante el entrenamiento. Tras el primer filtrado, el equipo investigador decidió eliminar las tareas que estaban diseñadas para el calentamiento o vuelta a la calma debido a que repercutía en la fiabilidad de los resultados. Por ello, finalmente se tomaron como válidas 126 unidades de análisis estadístico (tareas) que se llevaron a cabo durante las 25 sesiones de entrenamiento que realizaron durante los tres meses finales de la temporada.

\section{Instrumento y Material}

En el desarrollo de esta investigación se emplearon hojas de sesiones para registrar las tareas realizadas en el entrenamiento y el Sistema Integral para el Análisis de las Tareas de Entrenamiento, SIATE (Ibáñez et al., 2016).

\section{Variables}

Como variable independiente del estudio se tomó la SJ. Esta variable pedagógica se recodificó para agrupar las tareas en cinco categorías. La variable resultante se denominó Tipo de SJ, con las siguientes categorías: i) Situaciones sin oposición, ii) Situaciones individuales, iii) Situaciones reducidas en igualdad numérica, iv) Situaciones de superioridad numérica, v) Full Games.

- Situaciones sin oposición: Todas las tareas en las que no existe jugador con rol de defensa. Esta categoría agrupa las situaciones de juego de $1 \mathrm{x} 0,2 \mathrm{x} 0,3 \mathrm{x} 0,4 \mathrm{x} 0$, $\mathrm{nx} 0$.

Situaciones individuales: Son las tareas en las que tan solo existe un jugador con rol de ataque y un jugador con rol de defensa. Esta categoría solo recoge las situaciones de juego de $1 \times 1$.

- Situaciones reducidas en igualdad numérica (Small Sided Games en Igualdad, SSG-I): Son todas las tareas con un número reducido de practicantes y en las que existe igualdad entre los jugadores con fase de ataque y los de fase de defensa. Esta categoría recoge las situaciones de juego de $2 \times 2,3 \times 3,4 \times 4,5 \times 5$.

- Situaciones reducidas en superioridad numérica (Small Sided Games en Superioridad, SSG-S): Son todas las tareas con un número reducido de practicantes y en las que existe desigualdad entre los jugadores con fase de ataque y los de fase de defensa. Esta categoría agrupa las situaciones de juego de $2 \times 1,3 \times 1,3 \times 2,4 \times 1$, $4 \times 2,4 \times 3,5 x 1,5 x 2,5 x 3 y 5 x 4$.

- Full Games: Son todas las tareas en las que el número de practicantes es igual o muy próximo a la situación que se da en competición. Esta categoría contiene las situaciones de juego de 6x6 y 7x7.

Las variables dependientes de la investigación empleadas se agruparon en función del tipo. Como Variables Pedagógicas se emplearon: i) La presencia de Portero (Portero); ii) Fase de Juego; iii) Tipo de Contenido; iv) Medio

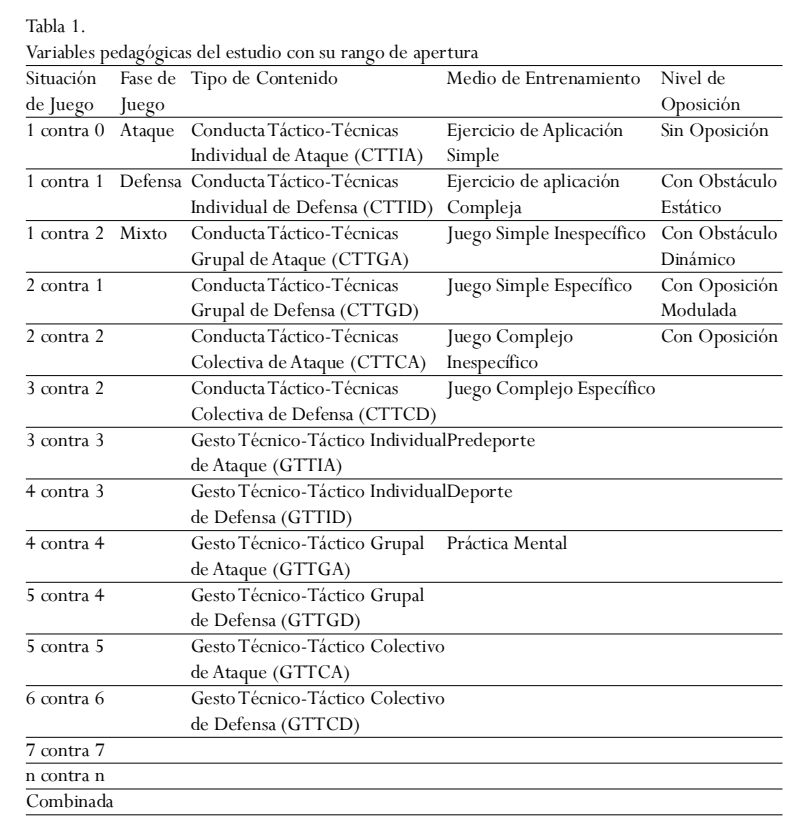


de Entrenamiento; v) Nivel de Oposición (Ibáñez et al., 2016). A continuación, se muestra la tabla 1 con las Variables Pedagógicas y las categorías de cada variable.

Las variables de carga externa (CE) subjetiva primarias seleccionadas para este estudio fueron: vi) Grado de Oposición; vii) Densidad de la Tarea; viii) Número de Ejecutantes Simultáneos; ix) Carga competitiva; x) Espacio de Juego; xi) Implicación Cognitiva. Las Variables secundarias de Carga Externa subjetiva seleccionadas fueron: xii) Carga Total de la Tarea; xiii) Carga Total ponderada por Segundos; xiv) Carga total por Minutos (Ibáñez et al., 2016). Todas las variables primarias están compuestas por cinco categorías. Estas categorías siguen un orden progresivo de complejidad, dificultad o requerimiento, otorgándole una puntuación de 1 a 5 puntos. Las variables secundarias se obtienen a partir de la manipulación de las variables primarias. La Carga Total de tarea se obtiene tras la suma de las puntuaciones de las seis variables primarias. La carga de una tarea tiene un rango mínimo de 6 puntos y máximo de 30 puntos. La Carga Total Ponderada por Segundos (CTPS) se obtiene multiplicando la carga total por el tiempo útil de práctica. Para reducir esta magnitud se genera una variable denominada Carga Total por Minutos en la que se divide la Carga Total Ponderada por Segundos entre 60 (Ibáñez et al., 2016). A continuación, se muestra la tabla 2 con las Variables de Carga Externa y las categorías de cada variable.

\begin{tabular}{|c|c|c|c|c|c|c|}
\hline Valor & Grado de Oposición & Densidad de & $\begin{array}{l}\text { Porcentaje de } \\
\text { Ejecutantes } \\
\text { Simultáneos }\end{array}$ & Carga Competitiva & Espacio de Juego & $\begin{array}{l}\text { Implicación } \\
\text { Cognitiva }\end{array}$ \\
\hline 1 & $\begin{array}{l}\text { Trabajo sin } \\
\text { Oposición } \\
\end{array}$ & $\begin{array}{l}\text { Ritmo Suave o } \\
\text { Trote }\end{array}$ & $0-20 \%$ & $\begin{array}{l}\text { Actividad sin } \\
\text { Competición } \\
\end{array}$ & $\begin{array}{l}\text { Lanzamientos de penalty / } \\
\text { sin desplazamiento }\end{array}$ & $\begin{array}{l}\text { Actividades sin } \\
\text { Relación }\end{array}$ \\
\hline 2 & $\begin{array}{l}\text { Trabajo de } \\
\text { Superioridad de } 3 \text { o } \\
\text { más deportistas } \\
\end{array}$ & $\begin{array}{l}\text { Ritmo Suave y } \\
\text { Continuo }\end{array}$ & $21-40 \%$ & $\begin{array}{l}\text { Concurso de Gestos de } \\
\text { Técnica }\end{array}$ & $\begin{array}{l}\text { Verticalidad Reducida / } \\
\text { máxima perpendicularidad } \\
\text { /1/4 de Campo }\end{array}$ & $\begin{array}{l}\text { Con Relación de } 1 \\
\text { comp/adversario }\end{array}$ \\
\hline 3 & $\begin{array}{l}\text { Trabajo de } \\
\text { Superioridad de } 2 \\
\text { deportistas } \\
\end{array}$ & $\begin{array}{l}\text { Intensidad con } \\
\text { Descanso }\end{array}$ & $41-60 \%$ & $\begin{array}{l}\text { Actividad con Oposición } \\
\text { sin contabilizar }\end{array}$ & 1/2 de Campo & $\begin{array}{l}\text { Con Relación de } 2 \\
\text { comp/adversario }\end{array}$ \\
\hline 4 & $\begin{array}{l}\text { Trabajo de } \\
\text { Superioridad de } 1 \\
\text { deportista }\end{array}$ & $\begin{array}{l}\text { Intensidad sin } \\
\text { Descanso }\end{array}$ & $61-80 \%$ & $\begin{array}{l}\text { Actividad Reducidas con } \\
\text { Oposición contabilizando } \\
\text { el resultado }\end{array}$ & Todo Campo & $\begin{array}{l}\text { Con Relación de } 3 \\
\text { comp/adversario }\end{array}$ \\
\hline 5 & $\begin{array}{l}\text { Situaciones de } \\
\text { Igual dad numérica }\end{array}$ & Alta Intensidad & $81-100 \%$ & $\begin{array}{l}\text { Partido con todas sus } \\
\text { Variantes }\end{array}$ & Todo Campo de ida y vuelta & $\begin{array}{l}\text { a Con Relación de } \\
\text { todo el equipo }\end{array}$ \\
\hline
\end{tabular}

do lugar, se analizaron los horarios de entrenamiento y se procedió a registrar todas las sesiones de manera ecológica ( $\sin$ interferir en el diseño ni desarrollo del entrenamiento). Finalizada la recogida de las sesiones de entrenamiento, se concertó una reunión con el entrenador para la confiabilidad de los resultados entre lo recogido por el analista y lo planificado por el entrenador. Seguidamente, se realizó el análisis estadístico y se aportó la información relevante al entrenador a modo de feedback y aportar un conocimiento científico y crítico sobre su planificación y metodología de entrenamiento con la finalidad de mejorar su calidad como entrenador.

Se procedió a la formación de un analista para la recogida de los datos a través de la herramienta SIATE. Tras su formación, se calculó la concordancia intra-observador a través del índice Multirater Kappa free (Randolph, 2005). Se obtuvo un coeficiente $K_{\text {free }} .083$, considerado como una fiabilidad casi perfecta según Landis y Koch (1977).

\section{Análisis estadístico}

Se realizó un análisis descriptivo para las variables cualitativas a partir de la frecuencia para conocer el número de veces que se ha producido cada una de las variables del estudio (Frecuencia y \%) y descriptivo (Media y Desviación Típica) para las variables cuantitativas. Seguidamente, se realizaron las pruebas de asunción de criterios a través del Supuesto de Normalidad (Kolmogorov-Smirnov), Supuesto de Homocedasticidad (Prueba de Levene) y supuesto de aleatorización (Prueba de Rachas), encontrando una distribución de los datos como No Paramétrico (Field, 2009). Además, se realizó un análisis de covariación
Las Variables Organizativas seleccionadas para la investigación fueron: xvi) Tiempo Total; xvii) Tiempo de Explicación; xviii) Tiempo Útil; xix) Aprovechamiento; xx) Asistentes; xxi) Participantes; xxii) Participación (Ibáñez et al., 2016).

\section{Procedimiento}

En primer lugar, se contactó con el club y los entrenadores para informarles sobre el proyecto a realizar. Una vez que se aceptó la propuesta, se hizo un consentimiento informado para los entrenadores y club con información relevante sobre la investigación. En segun- para poder conocer la existencia de relaciones entre variables $\left(\mathrm{Chi}^{2}\right)$ y su nivel de asociación ( $V$ de Cramer) y entre cuáles se producía dicha asociación. La interpretación del grado de asociación entre las variables se realizó mediante Residuos Tipificados Corregidos (RTC) $(>|1.96|)$ que proporcionan las tablas de contingencia (Field, 2009). Para identificar esta relación con las variables cuantitativas se empleó el coeficiente de correlación Rho de Spearman. También, se calculó la potencia observada (Õ) (Cárdenas, \& Arancibia, 2014). Para interpretar la potencia observada, se tomó como valores óptimos (>.80) (Cárdenas, \& Arancibia, 2014). Por último, se obtuvo la fuerza de la asociación de las varia- 
bles analizadas siguiendo la interpretación realizada por Crewson (2006): Insignificante (<.10); Baja (.10-.30); Moderada (.30-.50); Alta (>.50). La significatividad empleada fue de $p<.05$ (Field, 2009). El software empleado fue el SPSS 24.0 (SPSS Inc., Chicago, USA).

\section{Resultados}

En la tabla 3 se muestran los resultados pertenecientes a las variables pedagógicas de las tareas analizadas.

\begin{tabular}{|c|c|c|c|}
\hline \multicolumn{4}{|c|}{$\underline{\text { Resultados descriptivos de las Variables Pedagógicas }}$} \\
\hline & & $n$ & $\%$ \\
\hline \multirow{12}{*}{ SJ } & $1 \times 0$ & 23 & 18,30 \\
\hline & $1 \times 1$ & 5 & 4,00 \\
\hline & $2 \times 1$ & 2 & 1,60 \\
\hline & $2 \times 2$ & 15 & 11,90 \\
\hline & $3 \times 2$ & 7 & 5,60 \\
\hline & $3 \times 3$ & 15 & 11,90 \\
\hline & $4 \times 3$ & 22 & 17,50 \\
\hline & $4 \times 4$ & 21 & 16,70 \\
\hline & $5 \times 4$ & 2 & 1,60 \\
\hline & $5 \times 5$ & 7 & 5,60 \\
\hline & $6 \times 6$ & 6 & 4,80 \\
\hline & Combinada & 1 & 0,80 \\
\hline \multirow{3}{*}{ Fase de Juego } & Ataque & 70 & 55,60 \\
\hline & Defensa & 46 & 36,50 \\
\hline & Mixto & 10 & 7,90 \\
\hline \multirow{8}{*}{ Tipo de Contenido } & CTTID & 1 & 0,80 \\
\hline & GTTIA & 27 & 21,40 \\
\hline & GTTID & 4 & 3,20 \\
\hline & CTTGA & 29 & 23,00 \\
\hline & CTTGD & 30 & 23,80 \\
\hline & CTTCA & 18 & 14,30 \\
\hline & CTTCD & 15 & 11,90 \\
\hline & GTTCD & 2 & 1,60 \\
\hline \multirow{5}{*}{ Medio de Entrenamiento } & EAS & 30 & 23,80 \\
\hline & EAC & 90 & 71,40 \\
\hline & JSI & 2 & 1,60 \\
\hline & Predeporte & 2 & 1,60 \\
\hline & Deporte & 2 & 1,60 \\
\hline \multicolumn{4}{|c|}{$\begin{array}{l}\text { CTTID: Conductas Táctico-Técnico Individuales de Defensa; GTTIA: Gesto Técnico-Tácticos } \\
\text { Individuales de Ataque; GTTID: Gesto Técnico-Tácticos Individuales de Defensa; CTTGA: } \\
\text { Conductas Táctico-Técnico Grupales de Ataque; CTTGD: Conductas Táctico-Técnico Grupales } \\
\text { de Defensa; CTTCA: Conductas Táctico-Técnico Colectivas de Ataque; CTTCD: Conductas } \\
\text { Táctico-Técnico Colectivas de Defensa; GTTCD: Gestos Técnico-Tácticas Colectivas de } \\
\text { Defensa; EAS: Ejercicio de Aplicación Simple; EAC: Ejercicio de Aplicación Compleja; JSI: } \\
\text { Juego Simple Inespećíico. }\end{array}$} \\
\hline
\end{tabular}

Los resultados de la tabla 3 muestran que el entrenador diseña principalmente tareas con situaciones de $1 \mathrm{x} 1,4 \mathrm{x} 3$ y $4 \mathrm{x} 4$ con la intención de trabajar la fase de ataque, a través del trabajo de Conductas como contenido principal y mayoritariamente diseña las tareas como Ejercicios de Aplicación Compleja (EAC).

En la tabla 4 se muestran los resultados obtenidos de las Variables de Carga Externa subjetiva de las tareas analizadas, así como las Variables Organizativas cualitativas.

La tabla 4 muestra que el entrenador analizado plantea tareas con igualdad numérica $(55,60 \%$ de las tareas analizadas) entre los jugadores con fase de ataque y fase de defensa, planifica las tareas con una densidad de intensidad con descanso en el que participan la mitad o menos de los jugadores, siendo tareas con oposición sin contabilizar en un espacio de perpendicularidad reduci-
Tabla 4.

Resultados descriptivos de las Variables de Carga Externa subjetiva y Organizativas.

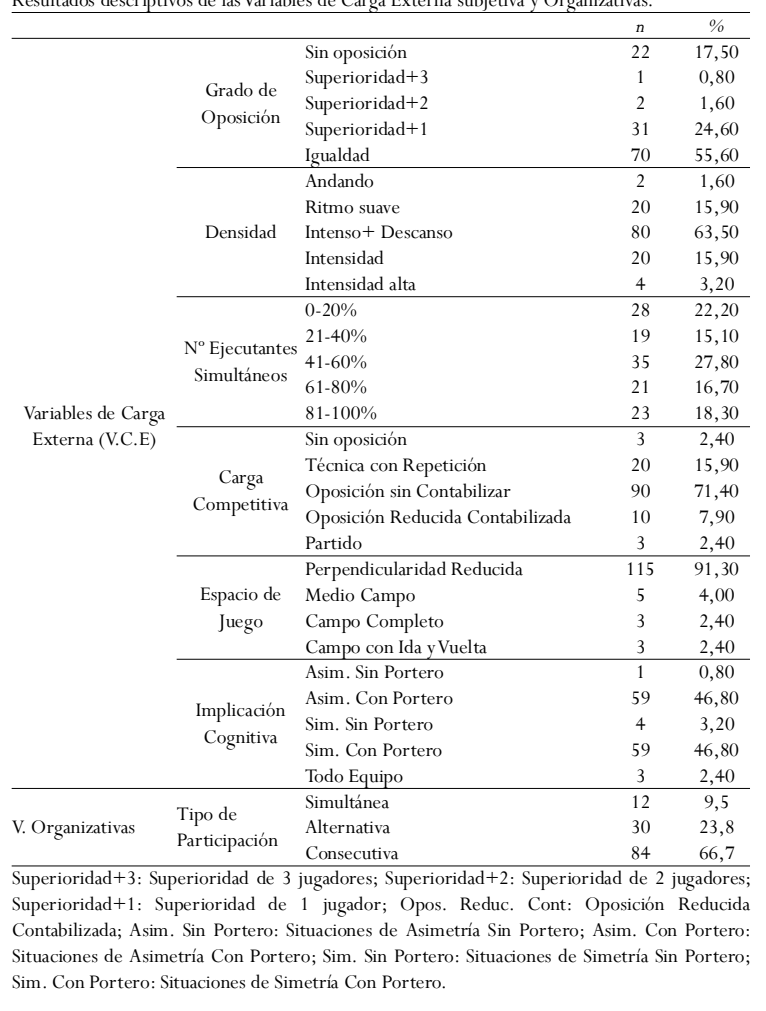

da (lateral-extremo) y en las que existe la presencia del portero. Además, el tipo de participación planteada en las tareas principalmente son con un diseño consecutivo.

En la tabla 5 se muestran los resultados de las Variables de Carga Externa secundarias, así cómo las organizativas cuantitativas.

\begin{tabular}{|c|c|c|c|}
\hline & & Media & $D T$ \\
\hline \multirow{4}{*}{$\begin{array}{c}\text { Variables de } \\
\text { Carga Externa } \\
\text { Subjetiva }\end{array}$} & Carga Total de la Tarea & 18,08 & 4,57 \\
\hline & Carga Total ponderada por Segundos & 8803,57 & 3904,13 \\
\hline & Carga Total ponderada por Segundos y Participación & 886,46 & 26,39 \\
\hline & Carga Total Minutos & 146,73 & 65,07 \\
\hline \multirow{7}{*}{$\begin{array}{c}\text { Variables } \\
\text { Organizativas }\end{array}$} & Tiempo Total & 518,10 & 145,32 \\
\hline & Tiempo de Explicación & 39,23 & 18,44 \\
\hline & Tiempo Útil & 478,87 & 138,20 \\
\hline & Aprovechamiento (\%) & 92,00 & 3,00 \\
\hline & Asistentes & 11,93 & 1,68 \\
\hline & Participantes & 5,85 & 3,27 \\
\hline & Participación (\%) & 49,03 & 3,98 \\
\hline
\end{tabular}

Los resultados de la tabla 5 muestran que las variables cuantitativas tienen una carga media de 18 puntos (en una escala entre 6 y 30). Además, el tiempo medio de las tareas diseñadas tienen una duración media de 520 segundos aproximadamente, siendo el tiempo de explicación inferior a los 40 segundos. Estos resultados confirman que el aprovechamiento del tiempo de entrenamiento es elevado (el 92\% del tiempo del entrenamiento, los jugadores están en movimiento). Por último, en cuanto a la participación de los jugadores en las tareas ronda el $50 \%$, esto explica que mientras la mitad de los jugadores ejecutan sus acciones en el en- 
trenamiento, la otra mitad está descansando o esperando a ejecutar.

En la tabla 6 se recogen el número de tareas diseñadas por el entrenador agrupadas por el Tipo de SJ. Los resultados muestran que la mayoría de las tareas son planteadas como Situaciones reducidas de igualdad numérica (Small Sided Games de Igualdad).

\begin{tabular}{lcc} 
Tabla 6. & & \\
Resultados descriptivos atendiendo al tipo de SJ. & $n$ & $\%$ \\
\hline Situación Sin Oposición & 23 & 18,3 \\
Situación Individuales & 5 & 4 \\
Small Sided Games de Superioridad Numérica & 34 & 27 \\
Small Sided Games de Igualdad Numérica & 58 & 46 \\
Full Games & 6 & 4,8 \\
\hline
\end{tabular}

A partir de los resultados de la tabla 6, se analiza la relación que tiene esta variable (Tipo de SJ) con el resto de las variables del estudio.

En la tabla 7 se muestran los resultados del análisis relacional entre el Tipo de SJ y las Variables Pedagógicas, de Carga Externa y Organizativas obtenido a través del Chi Cuadrado, $V$ de Cramer y Eta.

Tabla 7.

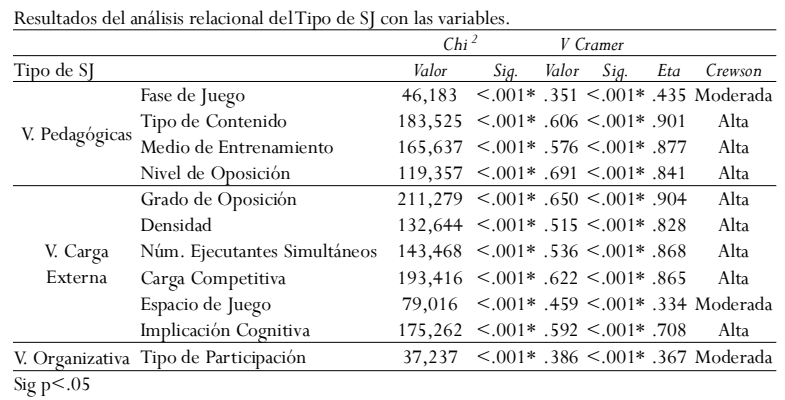

Se observa que todas las variables tienen una asociación estadísticamente significativa $(p<.05)$ con el Tipo de SJ. Además, la interpretación de estas relaciones ha sido contrastada con los ResiduosTipificados Corregidos (RTC) de las tablas de contingencia que deben ser superior a | 1.96| para que esta asociación exista. A continuación, se detallan las asociaciones existentes y el RTC que obtienen (Tabla 8). Los resultados positivos explican que, en las tareas analizadas, la asociación encontrada se da en mayor medida de las que inicialmente se podría esperar. Por el contrario, si el resultado del RTC es negativo, se han dado menor número de casos de los esperados inicialmente.

La tabla 9 muestra relación entre el Tipo de SJ y las Variables de Carga Externa y Organizativas cuantitativas del analizadas, mediante la prueba no paramétrica.

Tabla 9.

Resultados de la relación entre el tipo de SJ y la Variables de CE y Organizativas cuantitativas.

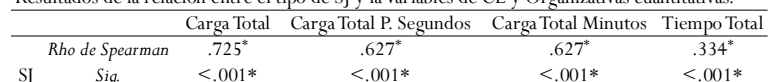

Tabla 8

Resultados de las relaciones contrastadas de las variables con la SJ a través de los RTC,

\begin{tabular}{|c|c|c|c|c|c|c|}
\hline \multirow{4}{*}{ Fase de Juego } & \multirow{2}{*}{$\begin{array}{l}\text { Tipo de SJ } \\
\text { Ataque }\end{array}$} & \multicolumn{4}{|c|}{ Sin Oposición Individuales SSG-I SSG-S } & \multirow[t]{2}{*}{$\mathrm{FG}$} \\
\hline & & 4.7 & & 3.1 & & \\
\hline & Defensa & -4.1 & & 3.6 & & \\
\hline & Mixto & & & & & 4.5 \\
\hline \multirow{8}{*}{$\begin{array}{l}\text { Tipo de } \\
\text { Contenido }\end{array}$} & CTTID & & 4.8 & & & \\
\hline & GTTIA & 10.4 & 2.2 & -5.3 & -3.4 & \\
\hline & GTTID & & & & & \\
\hline & CTTGA & -2.9 & & & & \\
\hline & CTTGD & -2.9 & & & & \\
\hline & CTTCA & & & & & 3.7 \\
\hline & CTTCD & & & 2.2 & & \\
\hline & GTTCD & & & & & \\
\hline \multirow{5}{*}{$\begin{array}{c}\text { Medio de } \\
\text { Entrenamiento }\end{array}$} & EAS & 9.4 & 4.1 & -5.4 & -3.3 & \\
\hline & EAC & -8.3 & -3.6 & 5.0 & 3.8 & \\
\hline & JSI & & & & & \\
\hline & Predeporte & & & & & \\
\hline & Deporte & & & & & \\
\hline \multirow{3}{*}{$\begin{array}{l}\text { Nivel de } \\
\text { Oposición }\end{array}$} & Sin Oposición & & & & -2.3 & \\
\hline & Con Oposición Modulada & & 2.5 & & & \\
\hline & Oposición & & & 4.6 & 2.0 & \\
\hline \multirow{5}{*}{$\begin{array}{l}\text { Grado de } \\
\text { Oposición }\end{array}$} & Sin oposición & & & & -3.1 & \\
\hline & Superioridad +3 & & & & & \\
\hline & Superioridad +2 & & & & & \\
\hline & Superioridad +1 & & & & 9.3 & \\
\hline & Igualdad & & & & & 2.3 \\
\hline \multirow{5}{*}{ Densidad } & Andando & 3.0 & & & & \\
\hline & Ritmo suave & 9.0 & & -4.5 & -2.9 & \\
\hline & Intenso + Descanso & -5.5 & & 3.1 & 3.0 & -3.3 \\
\hline & Intensidad & -2.3 & & & & 3.5 \\
\hline & Intensidad alta & & & & & 4.3 \\
\hline \multirow{5}{*}{$\begin{array}{l} \\
\mathrm{N}^{\circ} \text { Ejecutantes } \\
\text { Simultáneos }\end{array}$} & $0-20 \%$ & 9.9 & 2.1 & -5.2 & -3.1 & \\
\hline & $21-40 \%$ & -2.2 & & & & \\
\hline & $41-60 \%$ & -3.3 & & 2.5 & 3.1 & \\
\hline & $61-80 \%$ & -2.4 & & & & \\
\hline & $81-100 \%$ & -2.5 & & & & 5.3 \\
\hline \multirow{5}{*}{ Carga Competitiva } & Sin oposición & & & & & \\
\hline & Técnica con Repetición & 9.6 & & -4.5 & -2.9 & \\
\hline & aOpos. sin Contabilizar & -8.3 & & 5.0 & 3.4 & \\
\hline & Opos. Reduc. Cont. & & & & & \\
\hline & Partido & & & & & 7.8 \\
\hline \multirow{4}{*}{ Espacio de Juego } & Verticalidad Reducida & & & & & -5.4 \\
\hline & Medio Campo & & & & & \\
\hline & Todo Campo Completo & & & & & \\
\hline & Todo Campo con Ida y Vuelta & & & & & 7.8 \\
\hline \multirow{5}{*}{$\begin{array}{l}\text { Implicación } \\
\text { Cognitiva }\end{array}$} & Asim. Sin Portero & 5.6 & -2.2 & -8.4 & 6.7 & -2.4 \\
\hline & Asim. Con Portero & & & & & \\
\hline & Sim. Sin Portero & & & & & \\
\hline & Sim. Con Portero & -4.9 & 2.5 & 8.7 & -6.2 & \\
\hline & Todo Equipo & & & & & 7.8 \\
\hline \multirow{3}{*}{$\begin{array}{c}\text { Tipo de } \\
\text { Participación }\end{array}$} & Simultánea & & & & & 4.9 \\
\hline & Alternativa & -2.4 & & 3.0 & & \\
\hline & Consecutiva & 3.3 & & -2.5 & & \\
\hline
\end{tabular}

Individuales: Situaciones Individuales; SSG-I: Small Sided Games de Igualdad Numérica; SSG-S Small Sided Games de Superioridad Numérica; FG: Full Games; CTTID: Conductas Táctico Técnico Individuales de Defensa; GTTIA: Gesto Técnico-Tácticos Individuales de Ataque; GTTID: Gesto Técnico-Tácticos Individuales de Defensa; CTTGA: Conductas Táctico-Técnico Grupales de Ataque; CTTGD: Conductas Táctico-Técnico Grupales de Defensa; CTTCA: Conductas Técnico-Tácticas Colectivas de Ataque; CTTCD: Conductas Táctico-Técnico Colectivas de Defensa; GTTCD: Gestos Técnico-Tácticas Colectivas de Defensa; EAS: Ejercicio de Aplicación Simple; EAC: Ejercicio de Aplicación Compleja; JSI: Juego Simple Inespecífico; Superioridad +3: Superioridad de 3 jugadores; Superioridad+2: Superioridad de 2 jugadores Superioridad+1: Superioridad de 1 jugador; Opos. Reduc. Cont: Oposición Reducida Contabilizada; Asim. Sin Portero: Situaciones de Asimetría Sin Portero; Asim. Con Portero: Situaciones de Asimetría Con Portero; Sim. Sin Portero: Situaciones de Simetría Sin Portero; Sim. Con Portero: Situaciones de Simetría Con Portero.

\section{Discusión}

El objetivo de la presente investigación fue analizar el proceso de entrenamiento de un equipo de balonmano en categoria amateur. Los principales resultados confirman que el entrenador emplea situaciones reducidas y que la agrupación de jugadores influye en el resto de las variables que definen una tarea de entrenamiento, identificando una estructura estable de tareas de entrenamiento a partir del Tipo de SJ.

Se identifican relaciones en todas las variables analizadas en función del Tipo de SJ. Las agrupaciones de juego más empleadas por el entrenador analizado son las situaciones de SSG de igualdad y superioridad numéri- 
ca. En cuanto al análisis de esta variable, Cañadas, Ibáñez, García, Parejo, Feu (2013) confirmaron que a medida que se avanza de categoría, las agrupaciones de jugadores cambian aumentando el número de ejecutantes, siendo en categorías de formación las situaciones de $1 \mathrm{x} 0$ y 1x1 las más importantes. En esta investigación, los resultados obtenidos muestran que los mayores porcentajes se encuentran en situaciones $1 \mathrm{x} 0,4 \mathrm{x} 4,4 \mathrm{x} 3$. Estas diferencias se pueden deber a que el equipo analizado es de categoría senior y la etapa de formación del deportista ya ha finalizado y se prioriza el rendimiento por encima de la formación del jugador. La presencia elevada de situaciones de $1 \mathrm{x} 0$ puede estar motivada por la concepción metodológica del entrenador, en la que predomina el entrenamiento técnico para la mejora de gestos técnico-táctico individuales de ataque, como puede ser el lanzamiento. Por este motivo es tan importante el trabajo de tareas de finalización y efectividad en los lanzamientos. La situación de 4x3 (tarea en desigualdad numérica) supone un valor predictivo del resultado final de los partidos (Gutiérrez, Fernández, \& Borrás, 2010). Por otro lado, el trabajo en situación $4 x 4$ también es predictor del rendimiento, puesto que existe mayor implicación y percepción del esfuerzo en comparación con las situaciones 7x7 (Morales, \& Arias-Estero, 2015). La variedad en el empleo de diferentes agrupaciones en las tareas se puede deber a la etapa formativa de los jugadores y la experiencia previa que tenían. Los deportes de invasión se caracterizan por planificar el desarrollo integral del deportista a través de tareas para la formación individual y trabajo de patrones tácticos de juego (colectivos).

En cuanto a la asociación entre las Variables Pedagógicas se identificó una relación significativa con una intensidad moderada entre la Fase de Juego y la SJ. La asociación entre el Tipo de Contenido, Medio de Entrenamiento y Nivel de Oposición con el Tipo de SJ fue alta. En esta línea, Cañadas, Ibáñez, García y Feu (2012), realizaron un estudio sobre el análisis de las Variables Pedagógicas y afirmaron que una mayor variedad en el empleo de la SJ favorece una mejor formación del deportista. La importancia del empleo óptimo de estas variables reside en la mejora las diferentes formas de juego (Menezes, Reis y Morato, 2016; Feu, 2018), puesto que el entrenamiento técnico-táctico basado en situaciones tácticas proporciona un mayor desarrollo de la capacidad de jugar ante las situaciones problemáticas que pueden aparecer durante el contexto de un juego real (Morales, Greco y Andrade, 2009; Moreira, Matias y Greco, 2013; Dallegrave, Berno y Folle, 2017). Se identificó que el entrenador analizado utiliza agrupaciones de jugadores con un mismo patrón en función de la variable pedagógica que define la tarea de entrenamiento.

Los resultados en la Fase de Juego muestran una relación entre las tareas con fase de Ataque en tareas sin oposición o SSG de igualdad numérica. También, las tareas de SSG de igualdad numérica se emplean para el trabajo de la fase de Defensa, mientras que las tareas FG, tienen finalidad mixta. En línea con la Fase de Juego, Ibáñez (2002) explicó que las tareas de ataque siempre son más empleadas que las de defensa, aunque se debe intentar equiparar el número. Se identifica una predilección por diferentes agrupaciones de jugadores para entrenar contenidos de ataque o defensa, potenciando las tareas sin oposición para construir el juego de ataque y los SSG de igualdad numérica para entrenar los conceptos de ataque y defensa. La carga de entrenamiento provocada por las situaciones de defensa se ha identificado más alta que cuando se entrena el ataque (GarcíaCeberino, Gamero, Reina, Feu, \& Ibáñez, 2020). Este hecho está motivado por las variables primarias de carga externa subjetiva que condicionan la carga total de tarea de entrenamiento. La presencia de un mayor número de jugadores, con diferentes agrupaciones en los SSG, es uno de los factores para tener en cuenta (Mancha-Triguero et al., 2018; Gamero, García-Ceberino, Reina, Feu, \& Antúnez, 2020).

En cuanto al Tipo de Contenido, el empleo de Gestos Técnico-Tácticos tiene relación con las situaciones sin oposición. Esta es una estrategia habitual de entrenadores tradicionales posicionados en una metodología más mecanicista y también es identificado cuando el entrenador es novel o tiene poca formación (Cañada e Ibáñez, 2010). Sin embargo, en el resto del Tipo de SJ, a excepción de los SSG de superioridad numérica, se plantean mediante, es decir, el entrenador diseñó tareas en las que focalizó su atención sobre la solución táctica a un problema de juego. Esta modalidad es empleada en sesiones de entrenamiento bajo metodologías compresivas que centran el aprendizaje en el alumno/deportista (Ibáñez, Jiménez, \& Antúnez, 2015), priorizando en primer lugar sobre el qué hay que hacer (táctica) sobre el cómo hacer (técnica). El entrenador analizado tiene muy definida su forma de intervención, empleando las situaciones de juego sin oposición para el entrenamiento de los Gestos Técnico-Táctico de Ataque, las situaciones individuales para el entrenamiento de las Conductas Táctico-Técnicas de Defensa y en menor medida, de los Gestos Técnico-Táctico de Ataque. Finalmente, recurre al FG para el desarrollo de las Conductas Táctico- 
Técnicas de Ataque, tan importantes en los deportes de invasión (González-Espinosa et al., 2017).

Existe una asociación entre el Tipo de SJ y la variable Medio de Entrenamiento, identificándose que el entrenador planifica ejercicios de aplicación simple (EAS) para realizar tareas sin oposición o individuales, mientras que para las situaciones de SSG, emplea ejercicios de aplicación compleja (EAC). Los resultados han puesto de manifiesto un empleo excesivo de los ejercicios, simples o complejos en la forma de diseñar las tareas de entrenamiento. El empleo de Ejercicios, al igual que los Gestos Técnico Tácticos, está relacionado con metodologías tradicionales (Mancha-Triguero et al., 2021). Cañadas, Gómez, García-Rubio e Ibáñez (2018) afirmaron que en etapas en las que se fomenta el desarrollo cognitivo del deportista, el empleo de Juegos facilita este proceso de enseñanza-aprendizaje. El análisis de las tareas de entrenamiento ha identificado que la metodología del entrenador se aproxima más a modelos basados en la instrucción directa, que recuren a ejercicios para el entrenamiento de los contenidos de juego.

Finalmente, se han identificado asociaciones en el diseño de las tareas entre el Tipo de SJ y la Variable Pedagógica Nivel de Oposición. Emplea la oposición modulada en situaciones individuales mientras que la oposición es constante en las tareas en las que agrupa a los jugadores en situaciones reducidas. La manipulación del constreñimiento de la oposición es una de las estrategias que permite incrementar la carga de las tareas de entrenamiento (Ibáñez, Pérez-Goye, García-Rubio, \& CourelIbáñez, 2020). Las situaciones con oponentes, con independencia de su nivel de oposición, debe ser una constante en el entrenamiento en los deportes de invasión, pues permite mejorar las tomas de decisiones de los jugadores incrementando la carga de entrenamiento y asemejando las condiciones de práctica a la realidad deportiva.

En cuanto a las Variables de CE, se identificó una asociación entre el Grado de Oposición de las tareas diseñadas, empleando las situaciones de superioridad +1 en los SSG-D. El empleo de estos juegos reducidos con ventajas numéricas es habitual en el deporte del balonmano, pues se trata de reproducir una de las situaciones habituales que se producen en los partidos por las exclusiones. En esta línea, la similitud con la competición de las situaciones planteadas, facilitan la mejora del componente cognitivo y la motivación del deportista, puesto que repercute en el desarrollo de la libertad y creatividad (Giménez, \& Saénz-López, 2004) ante si- tuaciones tan importante en deportes con entornos cambiantes como es el balonmano.

Las tareas planteadas por el entrenador tienen una Densidad, intensidad, asociada a la agrupación de los jugadores. Mientras las intensidades son bajas en las tareas sin oposición, ésta se incrementa en los SSG en igualdad y desigualdad, así como en el juego colectivo. La carga de una tarea está condicionada por los objetivos de la tarea, el número de participantes, el espacio de juego, la presencia de portero entre otros factores (Ibáñez, Pérez-Goye et al., 2020). Mancha-Triguero et al., (2021) afirman que, en deportes de invasión como el baloncesto, la opción más empleada en entrenamientos es la categoría Intensidad sin descanso. En esta ocasión, el balonmano tiene un factor diferenciador al baloncesto y los cambios se realizan con mayor frecuencia, puesto que se diferencia entre jugadores para fase de ataque y jugadores para fase defensiva. Esta opción explica que el diseño de las tareas se realice a través de aplicar cargas intensas con descanso. En esta misma línea, se ha puesto de manifiesto que en el diseño de las tareas sin oposición este entrenador emplea organizaciones muy tradicionales con escasa participación (0$20 \%$ ), incrementándose la participación cuando las tareas se hacen más complejas siendo el FG el que reclama una mayor participación. El entrenador debe promover tareas con participación alta para que el jugador tenga mayor tiempo de práctica, sus vivencias sean mayores, y, por tanto, su aprendizaje y rendimiento físico se verá incrementado positivamente (GarcíaCeberino, Antúnez, et al., 2020).

El balonmano requiere de la interacción entre los compañeros y los adversarios, siendo necesarias tareas que impliquen una carga competitiva en el jugador al tener que atender a los estímulos provenientes de todos los jugadores. El entrenador diseña tareas sin oposición para trabajar la repetición de gestos técnicos, mientras que las situaciones con oposición sin contabilizar los resultados se emplean en las Situaciones de SSG de igualdad o superioridad numérica. La competición es empleada en la SJ de tipo FG y se lleva a cabo en el espacio de juego de campo con ida y vuelta. Se identifica un patrón estable en el diseño de tareas de entrenamiento. González-Espinosa et al., (2017) propusieron que el diseño de tareas de entrenamiento se asemejase a situaciones próximas a la competición, pudiendo realizar diferentes variantes que favoreciesen el desarrollo cognitivo y planteasen un reto motivante al deportista.

El espacio de juego empleado por el entrenador en sus tareas es variado, encontrándose sólo una relación 
con el empleo de todo el campo con idas y vueltas en ellos FG. Hill-Haas, Coutts, Rowsell y Dawson (2009) demostraron que las situaciones de juego reducidas (SSG) con un menor espacio provoca una mayor frecuencia cardíaca que las situaciones de juego con mayor número de componentes.

Las situaciones con portero son las más empleadas en los SSG de igualdad y superioridad numérica o todo el equipo para situaciones de FG favoreciendo la implicación cognitiva de los jugadores, a excepción de las situaciones sin oposición que se realizan sin presencia del portero. Estas elecciones son las más cercanas a las que encontraran los jugadores en competición. Por ello, el proceso enseñanza-aprendizaje del equipo, aun teniendo en cuenta la categoría en la que se encuentran, siguen trabajando aspectos técnico-tácticos para desarrollar habilidades en los jugadores García-Ceberino, Antúnez, et al., (2020). Además, en concordancia con los resultados obtenidos, el rendimiento de un equipo está vinculado a la incidencia en la actuación del portero, de ahí que sea de vital importancia en el equipo (Antúnez, 2003; Gómez-López, Sánchez, \& Antúnez, 2020; Román, 1995). El análisis de las tareas en las que se incluye la presencia del portero permite identificar parcialmente la metodología de entrenamiento empleada por el entrenador. El objetivo del deporte del balonmano es conseguir el gol. Para ello, los atacantes deberán superar una oposición directa (adversario) y otra indirecta (portero). Cuando los entrenadores emplean situaciones de juego sin presencia del portero, disminuyen la capacidad de toma de decisiones de los jugadores atacantes (Gómez-López, Sánchez, LópezTriviño, \& Antúnez, 2021). Identificar el número de tareas en las que existe el portero mejora la formación táctica de los jugadores.

En cuanto a la organización de las tareas se identifica un patrón de comportamiento claro. Se emplea práctica consecutiva para las tareas sin oposición, alternativa para trabajar con pequeños grupos y simultánea cuando es todo el equipo. La participación consecutiva repercute negativamente en la formación del deportista, puesto que el aprovechamiento del entrenamiento no es máximo, la intensidad es menor y no lleva asociadas implicaciones cognitivas al realizarse sin oponente. Con la participación alternativa y sobre todo con la consecutiva el aprovechamiento si es óptimo puesto que participan más los jugadores a la vez y el número de vivencias y practica es mayor, repercutiendo en la formación del deportista de manera positiva (González-Espinosa, Ibáñez, et al., 2017; González-Espinosa, Antúnez, Feu,
\& Ibáñez, 2018). El entrenador debe mejorar la organización de las tareas de entrenamiento reduciendo las organizaciones con práctica consecutiva para incrementar las experiencias del jugador.

Finalmente, se han identificado relaciones significativas en todas las variables de CE y Organizativas cuantitativas en función del Tipo de SJ. Estos resultados confirman que la elección de la agrupación de una tarea repercute en la Carga Total que soporta el deportista. Se ha puesto de manifiesto que existe margen para incrementar la carga de las tareas en las seis variables primarias que permiten obtener la carga de una tarea. Esta carga tiene un valor medio, sólo 18 puntos sobre 30 (Ibáñez, Pérez-Goye, et al., 2020). La interacción de este valor con el tiempo y la participación ofrece al entrenador una carga ajustada a la implementación de la tarea (García-Ceberino, Gamero, et al., 2020). En el caso analizado, las mayores agrupaciones de jugadores tienen una mayor carga teórica al obtener mayores puntuaciones en el grado de oposición, densidad, número de ejecutantes, carga competitiva espacio de juego e implicación cognitiva.

\section{Conclusión}

El análisis del proceso de entrenamiento es una competencia del entrenador de balonmano que debe seguir evolucionando para conocer con objetividad como es el proceso de entrenamiento. Este proceso no solo favorece la planificación y el proceso de enseñanza-aprendizaje, también ayuda al entrenador a tomar decisiones mediante el feedback que puede recibir del control y análisis de su planificación. A través de este proceso, conoce si las tareas diseñadas cumplen lo inicialmente planteado o si, por el contrario, emplea en exceso o defecto una variable del entrenamiento.

El proceso de entrenamiento analizado muestra relaciones significativas en las variables pedagógicas que definen una tarea de entrenamiento, la carga externa subjetiva y la organización de las tareas en función de la SJ. El análisis realizado muestra que la elección de la agrupación en la SJ repercute en la selección del resto de variables. Estas elecciones identifican patrones generales en el uso de las variables. El entrenador analizado tiene un patrón de entrenamiento muy definido.

Existen diferentes investigaciones que comparten el mismo instrumento utilizado en esta investigación, pero en otras poblaciones o deportes. Esta metodología de análisis de tareas de entrenamiento abre una posible línea de investigación futura en el campo del entrena- 
miento o escolar. El análisis del proceso de entrenamiento es un tópico de estudio que requiere pocos recursos, es fiable y válida y se realiza de manera ecológica puesto que no se interviene en el proceso planificado por el entrenador/profesor.

Las limitaciones de la investigación encontradas fueron las variables situacionales del equipo analizado (categoría y nivel competitivo) que condicionan la extrapolación de los resultados. Igualmente, sólo se pudo analizar el segundo período competitivo de una temporada. El proceso de codificación, aun siendo sencillo, requiere de formación específica de codificadores para una correcta fiabilidad de los datos, por lo que antes de la realización de una investigación se precisa de información.

Como prospectivas de futuro, sería interesante aumentar el número de sesiones analizadas, en diferentes categorías y sexos (como en otros deportes que ya existen estos análisis) con el objetivo de obtener información del proceso de enseñanza-aprendizaje y su evolución.

\section{Aplicaciones prácticas}

Las aplicaciones prácticas de este tipo de investigaciones tienen mayor peso en el campo del entrenamiento que en el científico. Permite aportar información para completar el ciclo de calidad de la intervención del entrenador, pues esta acción permitirá que el entrenador obtenga información sobre su planificación. Si desarrolla lo inicialmente planteado y cómo afecta un factor en el resto de los elementos que definen sus entrenamientos. Además, se puede conocer el proceso de enseñanza-aprendizaje de los jugadores, así como cuantificar la carga que soportan en cada tarea, sesión o periodo concreto de entrenamiento.

\section{Agradecimientos}

Esta investigación ha sido subvencionada parcialmente por la Ayuda a Grupos de Investigación (GR18170) de la Junta de Extremadura (Consejería de Economía e Infraestructuras); con la aportación de la Unión Europea a través de FEDER.

\section{Referencias}

Antúnez, A. (2003). La interceptacion en la portera de balonmano: efectos de un programa de entrenamiento perceptivo-motriz. Tesis Doctoral. Universidad de Mur- cia.

Bompa, T.O., \& Buzzichelli, C. (2018). Periodization: Theory and methodology of training. Champaing (USA): Human Kinetics.

Cañadas, M., \& Ibáñez, S.J. (2010). Planning the contents of training in early age basketball teams. E-Balonmano com, 6(1), 49-65.

Cañadas, M., Gómez, M.Á., García-Rubio, J., \& Ibáñez, S.J. (2018). Analysis of Training Plans in Basketball: Gender and Formation Stage Differences. Journal of Human Kinetics, 62(1), 123-134. https://doi.org/ 10.1515/hukin-2017-0164

Cañadas, M., Gómez, M.Á., García-Rubio, J., \& Ibáñez, S.J. (2018). Analysis of training plans in basketball: gender and formation stage differences. Journal of Human Kinetics, 62(1), 123-134.

Cañadas, M., Ibáñez, S.J., \& Leite, N. (2015). A novice coach's planning of the technical and tactical content of youth basketball training: A case study. International Journal of Performance Analysis in Sport, 15, 572-587.

Cañadas, M., Ibáñez, S.J., García, J., Parejo, I., \& Feu, S. (2013). Las situaciones de juego en el entrenamiento de baloncesto en categorías base. Revista Internacional de Medicina y Ciencias de la Actividad Física y el Deporte, 13(45), 41-54.

Cañadas, M., Ibáñez, S.J., García, J., Parejo, I., \& Feu, S. (2012). Estudio de las fases de juego a través del análisis del entrenamiento deportivo en categoría minibasket. Cuadernos de Psicología del Deporte, 12(2), 73-82.

Cárdenas, M., \& Arancibia, H. (2014). Potencia estadística y cálculo del tamaño del efecto en G* Power: Complementos a las pruebas de significación estadística y su aplicación en psicología. Salud \& Sociedad, 5(2), 210-224

Clemente, F.M., Martins, F.M.L., \& Mendes, R.S. (2015). How coaches use their knowledge to develop small-side soccer games: a case study. South African Journal for Research in Sport Physical Education and Recreation, 37(1), 1-11.

Cummins, C., Orr, R., O’Connor, H., \& West, C. (2013). Global positioning systems (GPS) and microtechnology sensors in team sports: A systematic review. Sports Medicine, 43(10), 1025 1042.

Dallegrave, E.J., Berno, C.S., \& Folle, A. (2017). Método situacional: Aplicação nos treinamentos técnico-táticos de uma equipe de base do handebol feminino. Corpoconsciência, 100-113.

Feu, S. (2018). El aprendizaje del balonmano en la edad 
escolar desde el Modelo Tactical Game. In S. Feu, J. García-Rubio, \& S.J. Ibáñez (Eds.), Avances científicos para el aprendizaje y desarrollo del Balonmano. Cáceres, España: Universidad de Extremadura. Servicios de Publicaciones.

Field, A. (2009). Discovering statistics using SPSS. Third Edition. Londres: SAGE.

Fox, J. L., Stanton, R., Sargent, C., Wintour, S. A., \& Scanlan, A.T. (2018). The association between training load and performance in team sports: A systematic review. Sports Medicine, 48(12), 2743-2774.

Gamero, M.G., García-Ceberino, J.M., González-Espinosa, S., Reina, M., \& Antúnez, A. (2017). Analysis of the Pedagogical variables in the tasks designed for the handball in pre-service teachers according to their gender. E-Balonmano com, 13(3), 217-224.

Gamonales, J.M., Gómez-Carmona, C.D., León, K., Muñoz-Jiménez, J., \& Ibañez, S.J. (2020). Study of the Pedagogical variables in grassroots football training tasks by competitive mesocycle. A case study. Retos, 37(37), 486-492.

García-Ceberino, J.M., Antúnez, A., Feu, S., \& Ibáñez, S.J. (2020). Quantification of Internal and External Load in School Football According to Gender and Teaching Methodology. International Journal of Environmental Research and Public Health, 17(1), 344. doi:10.3390/ijerph17010344.

García-Ceberino, J.M., Gamero, M.G., González-Espinosa, S., García-Rubio, J., \& Feu, S. (2018). Study of the external training load of tasks for the teaching of handball in pre-service teachers according to their gender. E-Balonmano com, 14(1), 45-54.

García-Ceberino, J.M., Gamero, M.G., Reina, M., Feu, S., \& Ibáñez, S.J. (2020). Study of External load in basketball tasks based on game phases. Retos. Nuevas Tendencias en Educación Física, Deporte y Recreación, (37), 540-545.

Giménez, F., \& Sáenz-López, P. (2004). El juego como medio en la enseñanza d baloncesto. En C. Jiménez, C. López y A. López (Eds.). II Curso de Didáctica del Baloncesto en las etapas de iniciación, 70-80.

Gómez-López, M., Sánchez, S.A., \& Antúnez, A. (2020). Efficiency of the goalkeepers in the women's World Handball Championship in Germany 2017. Revista Brasileira de Cineantropometria \& Desempenho Humano, 122, e72115.

Gómez-López, M., Sánchez, S.A., López-Triviño, J. \& Antúnez, A. (2021). Effectiveness of handball goalkeepers in the lastWord Championships for men and women. E-balonmano com, 17 (1), 13-22.
González-Espinosa, S., Antúnez, A., Feu, S., \& Ibáñez, S.J. (2018). Monitoring the external and internal load under two teaching methodologies. Journal of Strength and Conditioning Research, 34(10), 2920-2928.

González-Espinosa, S., Feu, S., García-Rubio, J., Antúnez, A., \& García-Santos, D. (2017). Diferencias en el aprendizaje según el método de enseñanza-aprendizaje en el baloncesto. Revista de Psicología del Deporte, 26, 65-70.

González-Espinosa, S., Ibáñez, S.J., y Feu, S. (2017). Design of two basketball Teaching programs in two different teaching methods. E-Balonmano com, 13(2), 131-152.

González-Espinosa, S., Mancha-Triguero, D., GarcíaSantos, D., Feu, S., \& Ibáñez, S.J. (2019). Diferencia en el Aprendizaje del Baloncesto según el Género y Metodología de Enseñanza. Revista de Psicología del Deporte, 28(3), 0086-92.

Gutiérrez, Ó., Fernández, J.J., \& Borrás, F. (2010). Ise of the effectiveness of the game Situations in inequality numerical in handball as predictive value of the final score. E-Balonmano com, 6 (2), 67-77.

Hill-Haas, S., Coutts, A., Rowsell, G., \& Dawson, B. (2009). Generic versus small-sided game training in soccer. International Journal of Sports Medicine, 30(3), 636-642.

Ibáñez, S.J. (2002). Los contenidos de enseñanza del baloncesto en las categorías de formación. En Ibáñez, S. y Macías, M. (Eds.), Nuevos horizontes para o treino do básquetbol. Cruz Quebrada: Facultade de Motricidade Humana.

Ibáñez, S.J. (2009). La intervención del entrenador de baloncesto: investigación e implicaciones prácticas. In A. Lorenzo, S. J. Ibáñez, \& E. Ortega (Eds.), Aportaciones teóricas y prácticas para el baloncesto del futuro (pp. 11-30). Sevilla: Editorial Wanceulen.

Ibáñez, S.J., Feu, S., \& Cañadas, M. (2016). Integral analysis system of training tasks, SIATE, in invasion games. E-Balonmano.com, 12(1), 3-30.

Ibáñez, S.J., García-Rubio, J., Antúnez, A., \& Feu, S. (2019). Coaching in Spain Research on the Sport Coach in Spain: A Systematic Review of Doctoral Theses. International Sport Coaching Journal, 6(1), 110125.

Ibáñez, S.J., Jiménez, A., \& Antúnez, A. (2015). Diferencias en las cargas de entrenamiento en baloncesto entre los modelos de enseñanza/entrenamiento comprensivo y técnico. Revista de Psicología del Deporte, 24(3), 47-50.

Ibáñez, S.J., Santos, J.A., \& García, J. (2015). 
Multifactorial analysis of free throw shooting in eliminatory basketball games. International Journal of Performance Analysis in Sport, 15(3), 897-912.

Ibáñez, S.J., Pérez-Goye, E., García-Rubio, J., \& CourelIbáñez, J. (2020). Effects of task constraints on training workload in elite women's soccer. International Journal of Sports Science \& Coaching, 15(1), 99-107.

Landis, J. R., \& Koch, G. G. (1977). An application of hierarchical kappa-type statistics in the assessment of majority agreement among multiple observers. Biometrics, 363-374.

Mancha-Triguero, D., García-Ceberino, J.M., Antúnez, A., \& García-Rubio, J. (2018). ¿Does the game phase affect the design of tasks for a formative basketball team? SPORT TK-Revista EuroAmericana de Ciencias del Deporte, 7(2), 27-36.

Mancha-Triguero, D., Reina, M., Feu, S., \& Ibáñez, S.J. (2021). Influencia del perfil del entrenador en el entrenamiento en baloncesto formativo influence of the coach's profile in formative basketball training. Revista Internacional de Medicina y Ciencias de la Actividad Física y del Deporte. In Press

Menezes, R.P., dos Reis, H.H.B., \& Morato, M.P. (2016). Team handball, unpredictability scenario and the methods of teaching-learning-training. EBalonmano com, 12(3), 165-176.

Montero, I., \& León, O.G. (2007). A guide for naming research studies in Psychology. International Journal of Clinical and Health Psychology, 7(3), 847-862.

Morales, J.C.P., Greco, P.J., \& Andrade, R.L. (2009). A description of the teaching-learning processes in bas- ketball and their effects on tactical procedural knowledge. Revista de Psicología del Deporte, 18(3), 469-473.

Morales, M., \& Arias-Estero, J.L. (2015). Differences between the 7 vs. 7 and 4 vs. 4 game handball at school in relation to performance, perceived exertion and intention to be physically active. Retos, $27,34-$ 39.

Moreira, V.J.P., Matias, C.J.A.D.S., \& Greco, P.J. (2013). A influência dos métodos de ensino-aprendizagemtreinamento no conhecimento tático processual no futsal. Motriz: Revista de Educação Física, 19(1), 84-98.

Oliver, J.F. (2018). El balonmano deporte de organización compleja. El error un componente de la auto organización del juego: una propuesta de entrenamiento. In S. Feu, J. Garcia-Rubio, \& S.J. Ibáñez (Eds.), Avances cientificos para el aprendizaje y Desarro110 del balonmano. Cáceres, España: Universidad de Extremadura. Servicios de Publicaciones.

Randolph J.J. (2005). Free-Marginal Multirater Kappa (multirater êfree): An Alternative to Fleiss' FixedMarginal Multirater Kappa. Joensuu Learning and Instruction Symposium, Joensuu, Finland.

Román, J.D. (1995). El portero. En Real Federación Española de Balonmano (Ed.), Clinics Deportes de Base y de Entrenadores de Élite: Madrid: RFEBM.

Urbano-Arévalo, F.J., Mancha-Triguero, D., Carmona, C., \& Gamonales, J.M. (2020). Influence of coach profile on the design of training tasks in initiation to football. A case study. Retos, 38(38), 204-212.

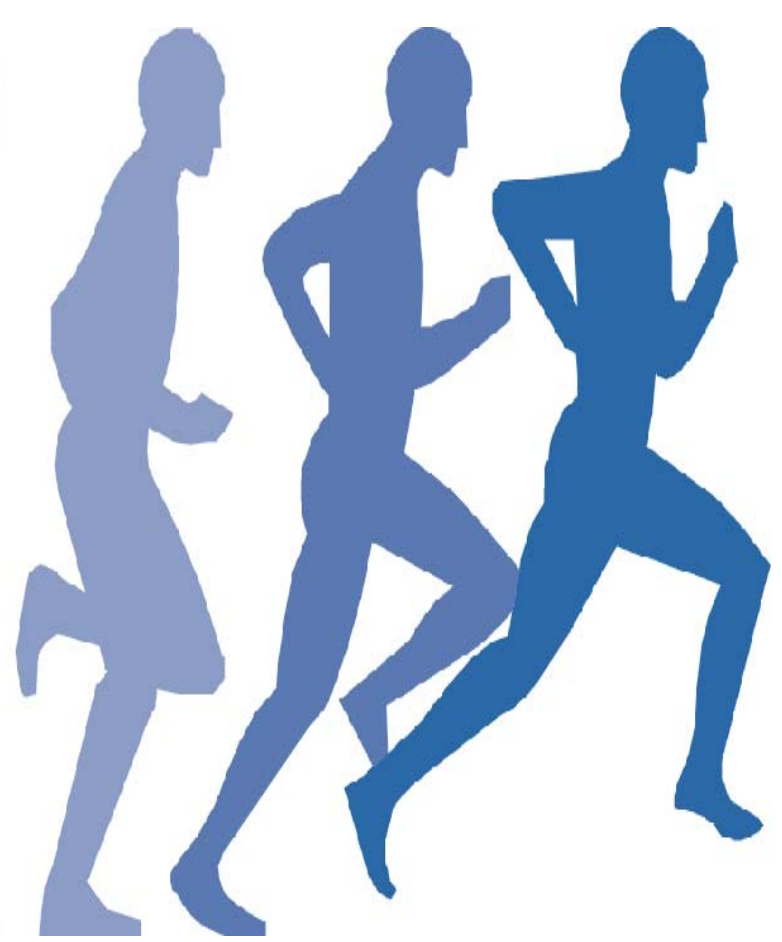

